\title{
Pronóstico de los pacientes recuperados tras un episodio de muerte súbita
}

\author{
R. RIDRUEJO, B. ZALBA, L. MARTÍN, A. CÁRCAMO \\ Servicio de Medicina Intensiva. Hospital Clínico Universitario Lozano Blesa. Zaragoza
}

\section{RESUMEN}

Objetivos: La muerte súbita constituye un grave problema con alta mortalidad y serias complicaciones neurológicas. El objetivo de este estudio es analizar el pronóstico y las características de pacientes inicialmente reanimados tras un episodio de muerte súbita y que son ingresados en Unidades de Cuidados Intensivos (UCI).

Método: Estudio retrospectivo de las características clínicas y estado al alta de 65 pacientes ingresados en UCI durante un periodo de 3 años tras una muerte súbita recuperada.

Resultados: 65 pacientes, $44(67,7 \%)$ hombres y $21(32,3 \%)$ mujeres. Edad media 69,1 $\pm 13,9.29(44,6 \%)$ muertes súbitas extrahospitalarias y 36 intrahospitalarias. La reanimación cardiopulmonar (RCP) fue $<10$ minutos en 28 casos $(43,1 \%), y>10$ minutos en $37(56,9 \%)$. Más de la mitad de los episodios tuvo origen cardiaco 55,4\% (36pacientes). 37 pacientes $(56,9 \%)$ fallecieron y $28(43,1 \%)$ sobrevivieron. Existió limitación del esfuerzo terapeutico (LET) en 11 casos (16,9\%). 29 pacientes $(44,6 \%)$ desarrollaron encefalopatía postanóxica (EPA) falleciendo la mayoría antes del alta. De los 28 supervivientes, 5 fueron dados de alta vivos con encefalopatía postanóxica $(17,8 \%)$ y 23 lo hicieron libres de secuelas neurológicas $(82,2 \%)$. Esto fué más frecuente cuando la parada cardiorrespiratoria (PCR) ocurrió dentro del hospital $(\mathrm{p} 0,009)$ y la reanimación duró menos de 10 minutos (p 0,045).

Conclusiones: Un elevado número de pacientes ingresados en UCI tras sufrir una PCR fallecen durante su estancia. Muchos desarrollan encefalopatía postanóxica y de ellos la mayoría muere. Pero a pesar de esto, hasta un 35\% de los pacientes admitidos tras una muerte súbita recuperada son dados de alta sin secuelas neurológicas.

PALABRAS CLAVE: Muerte súbita. Pronóstico. Encefalopatía postanóxica. Reanimación cardiopulmonar.

\author{
PROGNOSIS OF PATIENTS WHO RECOVERED AFTER AN EPISODE \\ OF SUDDEN DEATH
}

\begin{abstract}
Objectives: Sudden death constitutes a major sanitary problem with high mortality and serious neurological complications. The objective of this study was to analyze the prognosis and the characteristics of patients who initially recovered after an episode of cardiac arrest and who were admitted to the intensive care unit (ICU).

Method: We retrospectively studied the clinical characteristics and outcome of 65 patients admitted to the Intensive Care Unit during a 3 years period with aborted sudden death.

Results: 65 patients, 44 (67.7\%) men and 21 (32.3\%) women. Middle ages $69.1 \pm 13.9 .29(44.6 \%)$ out of hospital and 36 (55.4\%) into hospital sudden death. Cardiopulmonary resuscitation was $<10$ minutes in 28 cases $(43.1 \%)$, and $>10$ minutes in $37(56.9 \%) .36(55.4 \%)$ of all sudden deaths were of cardiac origin. 37 patients $(56.9 \%)$ died and $28(43.1 \%)$ survived the episode. It was LET in 11 cases (16.9\%). 29 (44.6\%) of all had post-anoxic encephalopathy and most died before discharge from ICU. Of 28 survivors, 5 patients were discharged alive with post-anoxic encephalopathy $(17.8 \%)$ and 23 were discharged without neurological disturbances $(82.2 \%)$. This was more frequent when sudden death was into hospital ( $p 0.009$ ) and cardiopulmonary resuscitation was $<10 \mathrm{~min}$ utes ( $p$ 0.045).

Conclusions: High number of the patients admitted to a Intensive Care unit with aborted sudden death died during ICU stay. Many patients had post-anoxic encephalopathy and most of these died. So, up to $35 \%$ of the patients admitted after an episode of cardiac arrest were discharged alive and without severe neurological damage.
\end{abstract}

KEY WORDS: Sudden death. Prognosis. Post-anoxic encephalopathy. Cardiopulmonary resuscitation.

Ridruejo R, Zalba B, Martín L, Cárcamo A, Montoiro R. Pronóstico de los pacientes recuperados tras un episodio de muerte súbita. An Med Interna (Madrid) 2007; 24: 217-220.

\section{INTRODUCCIÓN}

La muerte súbita constituye un problema sanitario de primer orden, ya que se estiman unos 370.000 paros cardiacos por año en toda Europa con una tasa de mortalidad que oscila del 65 al 95\% para las PCR extrahospitalarias y del 40 al 50\% para las intrahospitalarias (1).

Según el registro ICNARC de los pacientes que sobreviven menos de la mitad (45\%) serán dados de alta de las unidades de Intensivos y sólo el 30\% sobreviven al alta hospitalaria (2).

Trabajo aceptado: 15 de enero de 2007 
La encefalopatía postanóxica es una causa importante de morbimortalidad en pacientes reanimados que está en aumento en los últimos años debido a la generalización de programas de $R C P$ (3). Su frecuencia real es difícil de establecer, aunque según una encuesta realizada en 18 UCIS españolas en 1998 el diagnóstico de estado vegetativo figuraba en el 0,9\% de las altas (4).

Este problema supone una gran carga asistencial y consumo de recursos tanto materiales como humanos. Es por esto, que realizamos un estudio, con el objetivo de analizar las características y el pronóstico a corto plazo de pacientes que inicialmente son recuperados de una parada cardiorrespiratoria e ingresados en UCI.

\section{PACIENTES Y MÉTODO}

Se seleccionaron retrospectivamente 65 pacientes ingresados en la Unidad de Cuidados Intensivos del Hospital Clínico Lozano Blesa tras ser reanimados con éxito de un paro cardiorrespiratorio dentro ó fuera del hospital, durante un periodo de tiempo que abarca desde enero de 2002 a marzo de 2006.

Se analizaron los siguientes parámetros: a) datos demográficos (edad, sexo, antecedentes patológicos y estancia media en UCI); b) lugar del paro (intrahospitalario ó extrahospitalario), si este fue presenciado ó no y quién inició las maniobras de reanimación; c) tiempo de $R C P$ mayor ó menor de 10 minutos; d) etiología del paro; e) desarrollo ó no de encefalopatía postanóxica, que fue definida como la presencia de depresión del nivel de conciencia (Glasgow $<15$ ) tras $48 \mathrm{~h}$ del ingreso, en ausencia de medicación sedante, y diagnosticada clínicamente, aunque dicho diagnóstico fue apoyado en ocasiones por exploraciones complementarias (potenciales evocados somatosensoriales y EEG); y f) mortalidad y si existió limitación del esfuerzo terapeutico (LET).

Las variables cuantitativas se expresaron como media \pm desviación estándar (DE) y las cualitativas como porcentajes. La comparación de las variables cualitativas se realizó mediante el test de $\mathrm{Chi}^{2}$ y las cuantitativas con la U de MannWhitney considerando como estadísticamente significativos valores de $\mathrm{p}<0,05$. Para el análisis estadístico se utilizó el paquete estadístico SPSS 11.5.

\section{RESULTADOS}

De los 65 pacientes estudiados, $44(67,7 \%)$ eran hombres y $21(32,3 \%)$ mujeres, con edad media de $69 \pm 13,9$ años. En la tabla I se comparan las características clínicas, de atención a la parada y evolución de durante ingreso de los pacientes con y sin encefalopatía postanóxica.

Todos los pacientes fueron ingresados en UCI tras ser reanimados con éxito de una parada cardiorrespiratoria que ocurrió dentro del hospital en 36 ocasiones $(55,4 \%)$ y en 29 $(44,6 \%)$ fuera del hospital.

La primera atención fue dada por personal sanitario en la mayoría de los casos, 52 (80\%), siendo la familia, transeúntes $\mathrm{u}$ otras personas los primeros en socorrer en 13 ocasiones $(20 \%)$.

En 28 casos $(43,1 \%)$ el tiempo de resucitación duró menos de 10 minutos y en los 37 restantes las maniobras de $R C P$ se prolongaron más de 10 minutos.

\section{TABLA I}

CARACTERÍSTICAS CLÍNICAS, ATENCIÓN AL PARO Y EVOLUCIÓN DE LOS PACIENTES CON Y SIN ENCEFALOPATÍA POSTANOXÉMICA

\begin{tabular}{|c|c|c|c|c|}
\hline & No Pacientes & Sin epa (36) & Con epa (29) & $P$ \\
\hline Edad media $\pm D E$ & 65 & $69,6 \pm 14,8$ & $68 \pm 12,9$ & NS \\
\hline Sexo & 65 & & & \\
\hline Mujer & 21 & $10(47,6 \%)$ & $11(52,4 \%)$ & NS \\
\hline Varón & 44 & $26(59,1 \%)$ & $18(49,9 \%)$ & \\
\hline \multicolumn{5}{|c|}{ Antecedente cardiopat. isquémica } \\
\hline Sí & 22 & $14(63,6 \%)$ & $8(36,4 \%)$ & \multirow[t]{2}{*}{ NS } \\
\hline No & 43 & $22(51,2 \%)$ & $21(48,2 \%)$ & \\
\hline \multicolumn{5}{|c|}{ Antecedente enf. respiratoria } \\
\hline Sí & 10 & $5(50 \%)$ & $5(50 \%)$ & \multirow[t]{2}{*}{ NS } \\
\hline No & 55 & $31(56,6 \%)$ & $23(43,4 \%)$ & \\
\hline \multicolumn{5}{|c|}{ Antecedente enf. neurológica } \\
\hline Sí & 12 & $6(50 \%)$ & $6(50 \%)$ & \multirow[t]{2}{*}{ NS } \\
\hline No & 53 & $30(56,6 \%)$ & $23(43,3 \%)$ & \\
\hline \multicolumn{5}{|l|}{ Antecedente arritmias } \\
\hline Sí & 14 & $4(28,6 \%)$ & $10(71,4 \%)$ & \multirow[t]{2}{*}{ NS } \\
\hline No & 51 & $26(51 \%)$ & $25(49 \%)$ & \\
\hline \multicolumn{5}{|l|}{ Lugar de PCR } \\
\hline Extrehospitalaria & 29 & $11(37,9 \%)$ & $18(62,4 \%)$ & \multirow[t]{2}{*}{0,014} \\
\hline Intrahospitalaria & 36 & $25(69,4 \%)$ & $11(30,6 \%)$ & \\
\hline \multicolumn{5}{|l|}{ Primera atención } \\
\hline Familia/per. no sanitárico & 13 & $4(30,8 \%)$ & $9(69,2 \%)$ & \multirow[t]{2}{*}{0,063} \\
\hline Personal sanitárico & 52 & $32(61,5 \%)$ & $20(38,5 \%)$ & \\
\hline \multicolumn{5}{|l|}{ Duración RCP } \\
\hline$<10 \min$ & 28 & $23(82,1 \%)$ & $5(17,9 \%)$ & \multirow[t]{2}{*}{0,000} \\
\hline$>10 \min$ & 37 & $13(35,1 \%)$ & $24(64,9 \%)$ & \\
\hline \multicolumn{5}{|l|}{ LET } \\
\hline Sí & 11 & $2(18,2 \%)$ & $9(81,8 \%)$ & \multirow[t]{2}{*}{0,009} \\
\hline No & 54 & $34(63 \%)$ & $20(37 \%)$ & \\
\hline \multicolumn{5}{|l|}{ Exitus } \\
\hline Sí & 37 & $13(35,1 \%)$ & $24(64,9 \%)$ & \multirow[t]{2}{*}{0,000} \\
\hline No & 28 & $23(82,1 \%)$ & $5(17,9 \%)$ & \\
\hline Estancia media $\pm D E$ & 65 & $3,13 \pm 2,5$ & $8,2 \pm 10,5$ & 0,007 \\
\hline
\end{tabular}

PCR: parada cardiorrespiratoria; RCP: reanimación cardiopulmonar; DE: desviación estándar.

El origen más frecuente de la muerte súbita fue el cardiaco $36(55,4 \%)$ seguido del respiratorio $17(26,2 \%)$, neurológico 5 $(7,7 \%)$ metabólico $2(3,1 \%)$ y en 5 ocasiones no se consiguió saber la etiología del paro cardiorrespiratorio.

Del total de los 65 pacientes ingresados, $28(43,1 \%)$ sobrevivieron al episodio y $37(56,9 \%)$ fallecieron, siendo esto más frecuente en los 5 primeros días postparada (p 0,004). 29 (44,6\%) desarrollaron encefalopatía postanóxica, ocurriendo con más frecuencia cuando la $P C R$ fue extrahospitalaria ( 0 , 014) y cuando el tiempo de $R C P$ superó los 10 minutos (p $0,01)$. No encontramos asociación estadísticamente significativa con la edad ó la etiología del paro.

De los 28 supervivientes, 5 (7,7\% del total) fueron dados de alta con encefalopatía postanóxica y los 23 restantes $(35,38 \%)$ lo hicieron libres de secuelas neurológicas. Esto último fué más frecuente cuando el paro fue presenciado $(\mathrm{p}$ $0,045)$, si se produjo dentro del hospital $(\mathrm{p} 0,009)$ y cuando la $R C P$ duró menos de 10 minutos (p 0,000). 
Ninguna causa de PCR mostró una frecuencia significativamente mayor de muerte. Tampoco en la distribución por edad. Si bien el $64,9 \%$ de los fallecidos había presentado encefalopatía postanóxica (p 0,000$)$, existió LET en 11 ocasiones correspondiendo 8 de ellas a PCR extrahospitalarias ( $\mathrm{p}$ 0,051 ) y en 9 , a pacientes que habían desarrollado encefalopatía postanóxica (p 0,009).

\section{DISCUSIÓN}

Según observamos en los resultados de nuestro estudio la encefalopatía postanoxémica tras una parada cardiorrespiratoria es un hecho frecuente $(44,6 \%)$ y con elevada mortalidad $(82,75 \%)$, pero a pesar de ello hasta un $35,4 \%$ de los pacientes reanimados tras una muerte súbita, pueden ser dados de alta de UCI sin secuelas.

Estos resultados son difícilmente comparables, ya que el diseño de los distintos estudios publicados es diferente, por lo que en muchas ocasiones solo pueden verse semejanzas entre ellos (Tabla II).

En nuestro caso recogemos casos de muerte súbita intra y extrahospitalaria que ingresa en UCI, a diferencia de la mayoría de estudios, quienes sólo recogen paros extrahospitalarios (5-13). Esto puede crear algún sesgo a la hora de comparar, pues como demostramos, las $P C R$ intrahospitalarias desarrollan con menos frecuencia encefalopatía postanóxica ( $\mathrm{p}$ $0,014)$ y también en ellas es más frecuente el alta de UCI sin secuelas (p 0,009).

En el único estudio que hemos encontrado que no distingue el origen del paro (ICNARC) (2), de 22105 pacientes ingresados se consigue una supervivencia de UCI de $45 \%$, superponible a nuestros datos $43,1 \%$, aunque no se distingue si lo hacen con ó sin secuelas neurológicas.
Por otra parte del pequeño porcentaje de paradas extrahospitalarias que llega a ser ingresado en el hospital (entre 6,9 y $14,8 \%$ ) (7-8) del 50 al $79 \%$ fallecen durante la estancia hospitalaria (5-8). Nosotros desconocemos cuantos de los pacientes dados de alta de nuestra Unidad fallecieron posteriormente aunque un $56,9 \%$ lo hizo durante el ingreso en UCI, sin encontrar frecuencias significativas por edad a diferencia de otros estudios $(2,6,14-17)$ que atribuyen mayor mortalidad a la edad más avanzada.

Si analizamos la mortalidad según la existencia o no de secuelas neurológicas, esta es claramente más frecuente en pacientes que habían desarrollado EPA $($ p 0,000$)$ y durante los 5 primeros días de estancia (p 0,04). Posiblemente esta concentración de la mortalidad responde no solo a las complicaciones que este tipo de pacientes suele presentar, sino también a un menor uso de de medios diagnósticos y terapéuticos, incluido $L E T$, que es más frecuente en estos enfermos (p $0,009)$. Esto último se contempla en la última conferencia de consenso de la SEMICYUC atribuyendo un nivel de evidencia $\mathrm{C}$, grado de recomendación IIa a la decisión de LET cuando el paciente presenta ausencia de reflejo fotomotor, de respuesta motora al dolor y ausencia del componete N20 de los PESS al tercer día (4). Desconocemos si la hipotermía hubiera podido influir en un descenso de la mortalidad como en otros estudios (18-20) ya que dicha técnica ha comenzado a usarse recientemente en nuestro centro, con solo dos casos de los incluidos, uno de los cuales falleció y el otro fue dado de alta sin secuelas.

La presencia de secuelas neurológicas graves en los pacientes que sobreviven se ha relacionado con tiempos desde la parada ó reanimación, prolongados, superiores a 10 minutos como en nuestro en caso ( 00,000$)$ y en la mayoría de estudios publicados $(9-13,21-22)$. Esto apoya la decisión de no iniciar maniobras de reanimación cuando estas puedan ser inútiles ó insuficientes de cara a una recuperación satisfactoria

TABLA II

RESULTADOS DE DISTINTOS ESTUDIOS DE MUERTE SÚBITA

\begin{tabular}{|c|c|c|c|c|c|c|c|c|c|}
\hline Estudio & Procedencia & $\begin{array}{c}N^{0} \\
\text { Pacientes }\end{array}$ & $\begin{array}{l}\text { Tipo de muerte } \\
\text { súbita (extra, } \\
\text { intrahospitalaria } \\
0 \text { ambas) }\end{array}$ & $\begin{array}{l}\% \text { Pacientes } \\
\text { dados de } \\
\text { alta en UCl }\end{array}$ & $\begin{array}{l}\text { \% Pacientes } \\
\text { dados de } \\
\text { alta } \\
\text { hospitalaria }\end{array}$ & $\begin{array}{c}\% \text { Alta } \\
\text { hospitalaria } \\
\text { si secuelas }\end{array}$ & $\begin{array}{c}\% \text { Alta } \\
\text { hospitalaria } \\
\text { con secuelas }\end{array}$ & $\begin{array}{l}\text { \% Alta de UCI } \\
\text { con secuelas }\end{array}$ & $\begin{array}{c}\% \text { Alta de UCI } \\
\text { sin secuelas }\end{array}$ \\
\hline Cómez et al. & $\begin{array}{l}\text { Germán } \\
\text { Trias i } \\
\text { Pujol }\end{array}$ & 193 & M. Súb. Extra & - & $89(46 \%)$ & $51(26,3 \%)$ & $38(19,7 \%)$ & - & - \\
\hline Escorial et al. & $\begin{array}{l}\text { Gregorio } \\
\text { Marañón }\end{array}$ & 110 & M. Súb. Extra & - & $43(39 \%)$ & $33(30 \%)$ & $10(9 \%)$ & - & - \\
\hline $\begin{array}{l}\text { Martín Castro } \\
\text { et al }\end{array}$ & $\begin{array}{c}\text { Granada/Almería/ } \\
\text { Ejido }\end{array}$ & 282 & $\begin{array}{c}\text { M. Súb. Extra } \\
\text { RCP } 176 \\
\text { Ing. } 42(14,8 \%)\end{array}$ & - & $\begin{array}{c}9(21 \%) \\
0 \\
3 \% \text { del total }\end{array}$ & $\begin{array}{l}- \\
-\end{array}$ & $\begin{array}{l}- \\
-\end{array}$ & $\begin{array}{l}- \\
-\end{array}$ & $\begin{array}{l}- \\
-\end{array}$ \\
\hline$\overline{\mathrm{OPAL}}$ & Ontario & 5.335 & $\begin{array}{c}\text { M. Súb. Extra } \\
\text { Ing. } 366(6,9 \%)\end{array}$ & - & $\begin{array}{c}187(50 \%) \\
\text { ó 3,5\% del total }\end{array}$ & & & & \\
\hline NRCPR & $\begin{array}{c}207 \text { Hospitales } \\
\text { EE.UU. }\end{array}$ & 14.720 & M. Súb. Intra & - & $2.502(17 \%)$ & $\begin{array}{c}2.152 \\
(14,6 \%) \\
\end{array}$ & $\begin{array}{c}350 \\
(2,4 \%) \\
\end{array}$ & - & - \\
\hline ICNARC & Reino Unido & 22.105 & $\begin{array}{l}\text { M. Súb. } \\
\text { (ambas) }\end{array}$ & $9.974(45 \%)$ & $6.353(30 \%)$ & - & - & - & - \\
\hline Nuestro & $\begin{array}{l}\text { HCU Lozano } \\
\text { Blesa }\end{array}$ & 65 & $\begin{array}{l}\text { M. Súb. } \\
\text { (ambas) }\end{array}$ & $28(43,1 \%)$ & - & - & - & $5(7,7 \%)$ & $23(35,4 \%)$ \\
\hline
\end{tabular}


(23), aunque es un tema controvertido y en las últimas guías de la American Heart Association (24) se recomienda siempre el inicio de dichas maniobras.

A pesar de todo, hasta un $35,4 \%$ de los pacientes que han sufrido una PCR en nuestro medio salen de UCI sin secuelas y del hospital, según otras series del 14,6 al 30\% (5-6,25). Aún así, las tasas de EPA al alta continúan siendo elevadas ( nosotros $7,7 \%$ y otros estudios de 2,4 a $19,7 \%(5-6,25)$ por lo que es preciso incidir en los factores que pueden modificar esta evolución, especialmente el retraso de inicio de la RCP con programas adecuados y generalizados tanto a nivel poblacional, como al personal sanitario.

\section{Bibliografía}

1. Holzer M, Bernard S, Hachimi-Idrissi SA, Roine RO, Sterz F, Mullner M. Hypothermia for neuroprotection after cardiac arrest: Systematic review and individual patient data meta-analysis. Crit Care Med 2005; 33: 414-8.

2. Harrison DA, Brady AR, Rowan K. Case mix, outcome and length of stay for admissions to adult, general critical care units in England, Wales and Nothern Ireland: The Intensive Care National Audit and Research Centre Case Mix Programme Database. Crit Care 2004; 8: 99 111.

3. Marrugat J, Elosua R, Gil M. Epidemiología de la muerte súbita cardíaca en España. Rev Esp Cardiol 1999; 52: 717-25.

4. De La Cal MA, Latour J, de los Reyes M, Palencia E. Recomendaciones

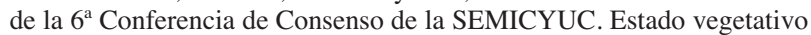
persistente postanoxia en el adulto. Med Intens 2003; 27: 544-55.

5. Gomez M,Serra J, Curós A, Claver E, Rodríguez Leor O, Bernal E, Valle V. Encefalopatía postanoxémica tras un episodio de muerte súbita cardiaca recuperada. Rev Esp Cardiol 2003; 56: 1064-8.

6. Escorial V, Meizoso T, Alday E, López de Sá E, Guerrero JE, LópezSendón JL. Pronóstico de los pacientes ingresados em la unidad coronária ó de cuidados intensivos trás un episodio de muerte súbita extrahospitalaria. Rev Esp Cardiol 2001; 54: 832-7.

7. Martín Castro C, Bravo M, Navarro Pérez P, Mellado Vergel FJ. Supervivencia y calidad de vida em la parada cardiorrespiratoria extrahospitalaria. Med Clin (Barc) 1999; 113: 121-3.

8. Stiell I, Wells G, De Maio V, Spaite D, Field B, Munkley D et al. Modificable factors associated with improved cardiac arrest survival in a multicenter basic life support/desfibrillation system: OPALS study phase I results. Ann Emerg Med 1999; 33: 44-50.

9. Laurent I, Monchi M, Chiche JD, Joly LM. Reversible myocardial dysfunction in survivors of out-of-hospital cardiac arrest. J Am Coll Cardiol 2002; 40: 2110-6.

10. Marin-Huerta E, Peinado R, Asso A, Loma A, Villacastín JP, Muniz J, et al. Muerte súbita cardíaca extrahospitalaria y desfibrilación precoz. Ver Esp Cardiol 2000; 53: 851-65.

11. Curós Abadal A. Parada cardiaca extrahospitalaria, nuestra asignatura pendiente. Rev Esp Cardiol 2001; 54: 827-30.

12. Kellermann A, Lynn J. Withholding Resuscitation in Prehospital Care. Ann Intern Med 2006; 144: 692-3.

13. Hillis M, Sinclair D, Butler G, Cain E. Prehospital cardiac arrest survi- val and neurologic recovery. J Emerg Med 1993; 11: 245-52.

14. Murphy DJ, Murray AM, Robinsi BE, Campion EW. Outcomes of cardiopulmonary resuscitation in the elderly. Ann Intern Med 1989; 11: 199-205.

15. Rogore HJ, Safar P, Sutton-Tyrrell K, Abramson NS. Old age does not negate good cerebral out-come alter cardipoulmonary resuscitation. Analysis from the brain resuscitation clinical trial. Crit Care Med 1995; 23: 18-25.

16. Van Alem AP, Waalewijn RA, Koster RW, de Vos R. Assessment of quality of life and cognitive function alter out-of-hospital cardiac arrest with successful resuscitation. Am J Cardiol 2004; 93: 131-5.

17. Fontanals J, Miró O, Pastor X, Grau JM, Torres A, Zavala E. Reanimación cardiopulmonar em enfermos hospitalizados em unidades convencionales. Estudio prospectivo de 356 casos consecutivos. Med Clin (Barc) 1997; 108: 441-5.

18. Hypothermia After Cardiac Arrest Study Group. Mild therapeutic hypothermia to improve the neurologic outcome after cardiac arrest. N Engl J Med 2002; 346: 549-56.

19. Efecto de la hipotermia moderada sobre el pronóstico de la encefalopatía postanóxica postparada cardiaca. Med Intens 2002; 26: 471-3.

20. Bernard SA, Gray TW, BuistMD, Jones BM, Silvester W, Gutteridge G, et al. Treatment of comatose survivors of out-of-hospital cardiac arrest with induced hypothermia. N Engl Med 2002; 346: 557-63.

21. Eisenberg M, Mengert T. Cardiac resuscitation. N Engl J Med 2001; 344: 1304-8

22. Herlitz J, Bang A, Gunnarson J, Engahl J. Factors associated with survival to hospital discharge among patiens hospitalised alive after out of hospital cardiac arrest: Change in outcome over 20 yerars in the community of Goteborg. Sweden. Heart 2003; 89: 25-30.

23. Curtis J, Park D, Krone M, Pearlman R. Use of the medical futility rationale in Do-Not Attempt-Resuscitation orders. JAMA 1995; 273: 124-8.

24. 2005 American Heart Association Guidelines for Cardiopulmonary Resuscitation and Emergency Cardiovascular Care. Circulation 2005; 112 (24 Supl.): IV-1-IV-5. (PMID: 16314375).

25. Peberdy MA, Kaye W, Ornato JP, Larkin GL, Nadcarni V, Mancini ME, Berg RA, Nichol G, Lane-Trultt T. Cardiopulmonary resuscitation of adults in the hospital: A reporto of 14,720 cardiac arrest from the National Registry of Cardiopulmonary Resuscitation. Resuscitation 2003; 58: 297-308. 